Case of curiosities

Brian Pippard

And Yet It Moves: Strange Systems and Subtle Questions in Physics. By Mark P. Silverman. Cambridge University Press: 1993. Pp. 266. £35, \$49.95 (hbk); £12.95, \$24.95 (pbk).

THIRTY years of living with physics have not dulled Mark Silverman's capacity to marvel at the incomprehensible weirdness of the world it describes, and when he finds yet another tantalizing example his instinct is to devise an experiment to make sure it is real. The result is a personal case-history, a series of investigations that a more stolid physicist (and there are many) might dismiss as wholly unnecessary; for if one accepts the standard formulation of quantum mechanics, its paradoxical consequences are inescapable and need no confirmation. Take the Aharanov-Bohm effect - the prediction that the interference pattern produced by splitting and recombining an electron beam may be shifted if magnetic flux threads through the space between the split beams, even though no magnetic field is allowed to reach the electrons themselves. If the effect were found not to occur, the whole structure of quantum mechanics would be undermined, but it was reasonably well verified long ago and I don't suppose Silverman expected to find otherwise but he had to be quite sure, and used the resources of modern electron microscopy to give an utterly convincing demonstration. Good for him.

This is only the preliminary to more complicated and taxing tests of old and new mysteries. Einstein-PodolskyRosen, Hanbury-Brown and Twiss, the Berry phase, the fifth force, Maxwell's demon and many others find their way into a wide-ranging and sometimes not fully articulated argument - Silverman cannot resist throwing in any little thing that fascinates him, even if it is only marginally relevant. Once this is recognized it is seen as part of the fun; and this is a book that is meant to be fun - it's written for those who haven't yet grown out of enjoying intellectual exercise.

Take warning, however; it is not easy reading. The absence of mathematics is no guarantee of a smooth ride - on the contrary, at this level of physics, mathematics is a positive aid, not an encumbrance. The words therefore have to be read very carefully, or the point of the argument slips away. Even with careful reading, the point sometimes eludes the grasp; for example, the discussion about reflection of polarized light by optically active materials is unconvincing because the alternative theories are not presented fully enough for one to understand what has been discovered by means of a very delicate measurement.

We have here a small book that could have been a much larger book if every topic had been given the detailed attention it deserves. But if it excites curiosity and leads the reader to think hard and to follow up the references for only one or two points, it will have done a useful job. The basic commodity the author offers for sale is his enthusiasm. He wants to share the adventure of tackling the deep intricacies of the physical world with as many others as possible. And it is greatly to his credit as a teacher that he offers no illusory short cuts.

Sir Brian Pippard is in the Cavendish Laboratory, University of Cambridge, Cambridge $\mathrm{CB} 3 \mathrm{OHE}$, UK.

\section{Getting down}

\section{Simon Klemperer}

A Continent Revealed: The European Geotraverse. Edited by Derek Blundell, Roy Freeman and Stephan Mueller. Cambridge University Press: 1992. Pp. 275. £35, $\$ 79.95$ (boxed set); £15.95, \$34.95 (paperback book only).

NEWTON claimed that he had seen a little further because he stood on the shoulders of giants. In the Earth sciences, still in equal parts descriptive and analytical, the giants' shoulders on which we stand today are not just prior scientific insights but also the data compilations that beget these insights. One such giant data-collection project has been the European Geotraverse (EGT), a seven-year international study of the lithosphere of the European continent, the results of which are summarized and synthesized in $A$ Continent Revealed. But this package is more than a scientific achievement. It is also a publishing tour de force, being part textbook, part research monograph, part geoscientific atlas and part digital dataset. It will be read and used in all these ways.

The EGT is one of the best examples of Earth-science collaboration in Europe. From 1983 to 1990 , scientists from 14 countries contributed to a series of experiments, particularly the collection of seismic data, to illuminate the threedimensional structure of a swath from northern Scandinavia to central Tunisia, a palimpsest of 3,500 million years of geological history. The territory studied is $4,600 \mathrm{~km}$ long (north to south), $250 \mathrm{~km}$ wide and, crucially to the philosophy of the EGT though sometimes only nominally in terms of achievement, $450 \mathrm{~km}$ deep. This emphasis on extending surface (and too often superficial) knowledge to depth is emblematic of one of the great achievements of modern Earth science. Only in the past three decades has it been possible - and only very recently routine - to probe significantly into the third dimension with precise seismic experiments. The EGT went beyond the scope of most national programmes to encompass nonseismic geophysics and geological mapping as well. Elsewhere in the world, only the Canadian Lithoprobe programme has obtained comparable interdisciplinary results.

The detailed scientific results of these experiments have already been published in eight special issues of the journal Tectonophysics, the proceedings of six workshops, and two dozen theses. What makes $A$ Continent Revealed special is the editors' achievement in summarizing a huge amount of primary scientific literature at a level accessible to an advanced undergraduate, and successfully melding the contributions of an additional dozen authors into a single narrative. But the main paperback book is not only a collection of facts; the authors are happily not afraid to attempt answers, however sketchy, to unsolved problems. This part of the package is available separately and will undoubtedly find use as a textbook; but in an attempt to give readers the same opportunity as the authors to interpret the database and to confront the scientific problems, the boxed set also contains 14 maps at a uniform scale of 1:2.5 million, a description of the data compiled in these maps and a CD-ROM containing some of the digital information incorporated into the maps. One can always ask for more for more of the original seismic data, or for digital versions of the maps suitable for a geographical information system - but the paper and digital databases already turn the textbook into a reference volume essential to any European geoscientist interested in the big picture.

For all that has been achieved, there is one telling omission. Despite the emphasis on imaging three-dimensional structure, not a single cross-section in $A$ Continent Revealed summarizes our new knowledge of the properties of the Earth as they vary with depth beneath the EGT. Despite seven years of data acquisition, such a cross-section would certainly have large gaps or uncertainties, and would show how far the geophysical community remains from the ideal of mapping the lower crust and mantle lithosphere in the same detail as we can map the Earth's surface. But how important, before we look forward from this giant's shoulders, to know where are its feet of clay; and how else to advance further than by acknowledging the sparseness of our data?

Simon Klemperer is in the Department of Geophysics, Stanford University, Stanford, California 94305-2215, USA. 